EXTENDED REPORT

\title{
Should oligoarthritis be reclassified? Ultrasound reveals a high prevalence of subclinical disease
}

\author{
R J Wakefield, M J Green, H Marzo-Ortega, P G Conaghan, W W Gibbon, D McGonagle, \\ $S$ Proudman, P Emery
}

Ann Rheum Dis 2004;63:382-385. doi: 10.1136/ard.2003.007062

See end of article for authors' affiliations

Correspondence to: Professor P Emery, Academic Department of Musculoskeletal Medicine, 1 st Floor, Old Nurse's Home, General Infirmary at Leeds, Great George Street, Leeds LSI 3EX, UK; p.emery@leeds.ac.uk

Accepted

9 September 2003
Objective: To determine the prevalence of subclinical synovitis using ultrasound (US) imaging of both painful and asymptomatic joints, in patients with early ( $<12$ months), untreated oligoarthritis ( $\leqslant 5$ joints). Methods: Eighty patients underwent a detailed clinical assessment by two physicians. All painful joints were identified, which were immediately scanned by a sonographer. In the last 40 patients, an additional standard group of joints was scanned to establish the prevalence of synovitis in asymptomatic joints.

Results: In 80 patients, 644 painful joints (with and without clinical synovitis) were identified and each underwent a US assessment. Of these joints, 185 had clinical synovitis, of which, US detected synovitis in only 79\% (147/185). In the other 38 joints US demonstrated tenosynovitis instead of synovitis in 12 joints and possible, but not definite, synovitis in 11 joints. Fifteen joints were, however, normal on US. In 459 joints that were not clinically synovitic, US detected synovitis in 33\% (150/459). In 64\% (51/80) of patients, US detected synovitis in more joints than clinical examination and in $36 \%$ (29/80) of patients, US detected a polyarthritis (>6 joints). Of the 826 asymptomatic (non-painful) joints scanned, 13\% (107) 826) had US detected synovitis.

Conclusion: Sonography detected more synovitis than clinical examination in patients with oligoarthritis. In almost two thirds of patients there was evidence of subclinical disease while one third could be reclassified as polyarticular. These findings suggest that a definition of oligoarthritis based purely on clinical findings may be inappropriate, which may have important implications for disease management.
$\mathrm{O}$ ligoarthritis is an inflammatory arthritis characterised by clinical swelling of only a few joints. Definitions are varied and range from two to four joints ${ }^{1}$ or less than six. ${ }^{2}$ The term encompasses a group of diseases including reactive arthritis, psoriatic arthritis, and undifferentiated arthritis, ${ }^{3}$ in addition to rheumatoid arthritis (RA) in evolution. Data on outcome in these patients demonstrate a variable rate of persistence after conventional treatment. ${ }^{45}$ Currently, no standardised treatment protocol exists, although intra-articular corticosteroids have recently been shown to be effective in a proportion of patients. $^{2}$

Ultrasonography (US) is an increasingly used technique by clinicians for the evaluation of inflammatory joint diseases. $^{6-10}$ It has been shown to be sensitive in the detection of synovitis and bone erosion in both small and large joints. ${ }^{1-18}$ It has several advantages over magnetic resonance imaging (MRI), including cost, immediate availability in the clinic, and the ability to scan multiple joints at one time.

One reason for the variability in outcome in patients with oligoarthritis is the heterogeneity of the population given that the diagnosis is based only on the number of joints with clinical synovitis. The prevalence of subclinical disease in these patients is unknown and may qualitatively alter subsequent outcome as well as defining a group of patients requiring more aggressive treatment.

This study aimed at determining the prevalence of subclinical synovitis defined by US in patients with early, untreated oligoarthritis. The ultrasonographer, unaware of the clinical findings, scanned all painful joints. Additionally, in the final 40 patients, a standard set of joints was scanned to determine the prevalence of synovitis in asymptomatic joints.

\section{METHODS}

\section{Patients}

This study received Leeds General Infirmary ethical committee approval and all patients gave written informed consent. Patients with early oligoarthritis ( $<12$ months symptoms) were recruited consecutively from the early arthritis clinics within the Yorkshire region. Oligoarthritis was defined as the presence of clinical synovitis in $\leqslant 5$ joints. Clinical synovitis required the fulfilment of at least two of the following three criteria: swelling, tenderness, or decreased range of movements of any peripheral joint (excluding a distal interphalangeal joint). The last criterion allowed the inclusion of the deeper joints such as the shoulder, where the usual signs of synovitis, in particular swelling, are difficult to elicit. All patients had stopped their non-steroidal anti-inflammatory drugs three days before entry and all were corticosteroid and disease modifying antirheumatic drug naïve.

\section{Ultrasound evaluation}

Ultrasonography was performed with an ATL HDI 3000 machine with a 10-5 MHz linear "hockey stick" transducer (Advanced Technologies Laboratories, Bothel, Washington, USA). All scans were performed by a rheumatologist (RJW) specifically trained in joint sonography. Gel was applied to the skin to provide an acoustic interface.

\footnotetext{
Abbreviations: $M C P$, metacarpophalangeal; $M R I$, magnetic resonance imaging; MTP, metatarsophalangeal; RA, rheumatoid arthritis; RF, rheumatoid factor; US, ultrasound
} 


\begin{tabular}{ll}
\hline \multicolumn{2}{l|}{ Table 1 Patient characteristics } \\
\hline Characteristics \\
\hline No of patients \\
Age (years), mean (range) & 80 \\
Women, No (\%) & $37.4(20-81)$ \\
Disease duration (weeks), mean (range) & $39(49)$ \\
Monarthritis, No (\%) & $18(2-52)$ \\
Number of swollen joints, mean (range) & $26(33)$ \\
CRP $>100 \mathrm{~g} / \mathrm{l}$, No (\%) & $2(1-5)$ \\
RF $>40$ IU/l, No (\%) & $54(68)$ \\
DR4 and/or DR1, No (\% of tested) & $12(15)$ \\
HLA-B27, No (\% of tested) & $47 / 71(66)$ \\
\hline RF, rheumatoid factor; CRP, C reactive protein. \\
\hline
\end{tabular}

\section{Definition of ultrasonographic synovitis and method of scoring}

Synovitis was defined as the presence of an abnormally hypoechoic joint space reflecting synovial hypertrophy, distinct from the intra-articular fat pad and non-compressible with the transducer. Synovial fluid was detected by the presence of an abnormally anechoic space within the joint, which was compressible. Joint examination technique followed the EULAR guidelines, ${ }^{19}$ and all findings were interpreted using both longitudinal and transverse planes. Synovitis was scored as either definitely present (1) or absent (0). The presence of bone erosions (cortical defect seen in two or more scanning planes), tenosynovitis (abnormally hypoechoic area around tendon seen in longitudinal and transverse planes), or other soft tissue abnormalities was also documented.

\section{Study design}

At presentation, each patient underwent a detailed clinical history and examination to identify all joints, which were currently $(<1$ week) painful (symptomatic). Two clinicians (MJG, PE) then examined these joints for the presence of clinical synovitis as previously defined and a consensus was reached between them. The clinical assessment was followed by a general laboratory screen including full blood count, erythrocyte sedimentation rate, plasma viscosity, $\mathrm{C}$ reactive protein, biochemical profile, rheumatoid factor (RF), antinuclear antibody, urate, immunoglobulin and HLA-DRB $1 * 01$ / DRB ${ }^{*} 04$ and HLA-B27 as well as appropriate microbiological and radiological investigations (including radiographs of the chest, hands, and feet in all patients).

A list of all painful joints was then given to the sonographer (RJW) who immediately scanned each joint for the presence of definite synovitis. After scanning the first 40 patients it became apparent that subclinical disease was very common and we therefore questioned whether synovitis might occur in asymptomatic joints. The protocol was therefore subsequently modified so that in the final 40 patients, a standard group of 22 joints was scanned (10 metatarsophalangeal (MTP) joints, 10 metacarpophalangeal

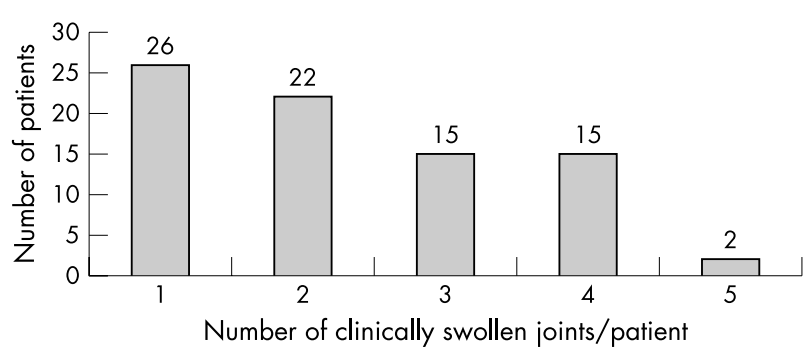

Figure 1 Number of patients ( $n=80)$ with swollen joints $(n=185)$ as detected by clinical examination.

(MCP) joints, 2 knees), irrespective of symptoms or signs, in addition to all symptomatic joints. The sonographer was unaware of which joints were either symptomatic or asymptomatic.

\section{Statistics}

The number of joints with clinical synovitis or US detected synovitis was not normally distributed and therefore the means, medians, and ranges were reported. Differences between patients with subclinical and no subclinical disease were measured by either the $\chi^{2}$ test or Fisher's exact test, as appropriate.

\section{RESULTS}

Eighty patients were recruited into the study. Table 1 gives the patient demographic data.

\section{Clinical findings}

In total, 1470 joints were clinically examined, of which 644/ $1470(44 \%)$ were painful and 826/1470 (56\%) were asymptomatic (non-painful and not swollen). Clinical synovitis was found in 185/1470 (12.6\%) joints examined: knee joints 52/ $185(28 \%)$, MTP joints 29 (16\%), MCP joints 21 (11\%), proximal interphalangeal (PIP) joints 21 (11\%), ankles 19 ( $10 \%)$, wrists $18(10 \%)$, elbows $5(3 \%)$, shoulders $3(2 \%)$, miscellaneous other joints $17(9 \%)$. Figure 1 shows the number of clinically swollen joints/patient. The mean and median number of clinically synovitic joints/patient was 2 (range 1-5). Twenty six patients had a monarthritis (14 knees, 8 ankles, 4 wrists), 52 patients had synovitis in two to four joints, but only two patients had synovitis in five joints.

\section{Ultrasound findings}

The mean and median number of joints/patient with US synovitis was five and four joints respectively (range 1-28). Figure 2 shows the frequency distribution of the number of joints with US detected synovitis. The frequency of joint types with US synovitis $(n=404)$ was: MTP joints $195(48 \%)$, MCP joints $65(16 \%)$, knees 65 (16\%), wrists $30(7 \%)$, ankles 22 (5\%), PIP joints $25(6 \%)$. US detected synovitis was found in 32/35 (91\%) clinically swollen MTP joints.

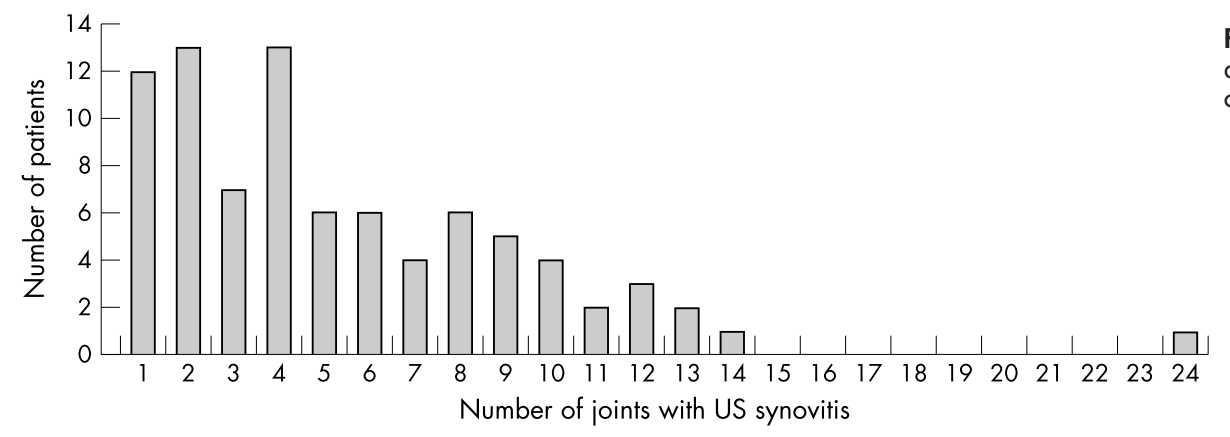

Figure 2 Number of patients with US detected synovitis in different numbers of joints. 


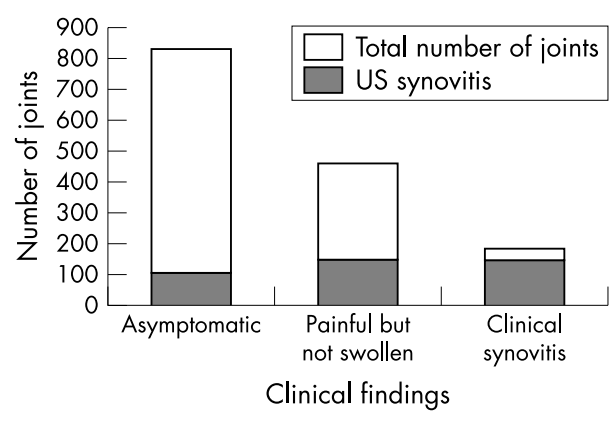

Figure 3 Prevalence of US detected synovitis in joints which were asymptomatic $(n=826)$, clinically painful but not swollen $(n=425)$, and clinically synovitic joints $(n=185)$.

\section{US synovitis in symptomatic joints (fig 3)}

US documented synovitis in 147/185 (79\%) clinically synovitic joints. All clinically synovitic joints were painful. In the other joints that were painful but not clinically synovitic, US detected synovitis in 150/459 (33\%) joints (fig 4).

Clinical examination detected more synovitis than US in 38 joints because US demonstrated tenosynovitis instead of synovitis in 12 joints ( 6 wrists, 3 ankles, 3 MCP joints) and possible, but not definite, synovitis in 11 joints ( 3 wrists, 3 ankles, 2 knees, 3 MTP joints). However, 15 joints with clinical synovitis were recorded as normal on US (4 wrists, 4 ankles, 3 knees, 3 MTP joints, 1 interphalangeal (IP) joint). This change in joint number, however, did not equate to a change in the number of patients with synovitis. Of the 26 patients who had a monarthritis based on clinical examination, $11 / 26(42 \%)$ corresponded exactly with US, 9/26 (35\%) had US synovitis in $>1$ joint (2-12), and 6/26 (23\%) had US synovitis defined polyarthritis ( $\geqslant 6$ joints).

\section{US detected synovitis in asymptomatic joints (fig 3)}

In total, $826 / 1470(56 \%)$ of joints scanned were clinically asymptomatic (non-painful and not swollen). Of these clinically normal joints, $13 \%(107 / 826)$ had evidence of subclinical synovitis. Of the 107 asymptomatic joints, which had US detected subclinical disease, 85 (79\%) were MTP joints, 17 (16\%) MCP joints, and 5 (5\%) knees. Only seven patients had subclinical disease in one additional joint, which consisted of 5 MTP joints (4 were a lst MTP joint) and 2 knees.

\section{Characteristics of patients with subclinical disease}

Table 2 gives the patient characteristics of those with and without subclinical disease. No statistically significant differences in sex, age, CRP, RF, DR4 and/or DRl, or
Table 2 Comparison of demographic and laboratory data between patients with or without subclinical disease

\begin{tabular}{|c|c|c|c|}
\hline & $\begin{array}{l}\text { Subclinical } \\
\text { disease } \\
(n=51)\end{array}$ & $\begin{array}{l}\text { No subclinical } \\
\text { disease } \\
(\mathrm{n}=29 \text { ) }\end{array}$ & Significance \\
\hline Female sex, No (\%) & $19 / 51$ (37) & $20 / 29(67)$ & NS \\
\hline Age (years), mean & 36 & 38 & NS \\
\hline $\begin{array}{l}\text { CRP }>100 \mathrm{~g} / \mathrm{l}, \text { No (\% of } \\
\text { tested) }\end{array}$ & $34 / 51$ (67) & $20 / 29(67)$ & NS \\
\hline $\begin{array}{l}\mathrm{RF}>40 \mathrm{IU} / \mathrm{I}, \text { No (\% of } \\
\text { tested) }\end{array}$ & $10 / 51(20)$ & $2 / 29(7)$ & NS \\
\hline DR4 and/or DR1, No (\%) & $26 / 51(51)$ & $21 / 29(72)$ & NS \\
\hline HLA-B27, No (\%) & $15 / 51(29)$ & $3 / 29(10)$ & NS \\
\hline
\end{tabular}

HLA-B27 status existed between the groups, although there was a trend towards those with subclinical disease being more likely to be RF or HLA-B27 positive. The prevalence of subclinical and polyarticular disease was also assessed according to baseline diagnosis (table 3). For the patient groups, 18 patients in the first 40 patients had subclinical disease and 33 in the second set of 40 . This may reflect the greater number of joints scanned in the second group (997 joints versus 473).

\section{DISCUSSION}

This study highlights for the first time, a high prevalence of subclinical synovitis defined by US in patients with early, untreated oligoarthritis and the relative insensitivity of clinical examination. It suggests that two thirds of patients have subclinical disease and about one third could be reclassified as having a polyarticular disease. In painful joints, US detected synovitis in $79 \%$ of those with clinical synovitis and $33 \%$ joints without synovitis. The prevalence of synovitis in asymptomatic joints was $13 \%$.

Ultrasound represents a safe and relatively inexpensive tool for joint examination and is ideally suited for multiple joint assessment. In contrast, traditional MRI is more expensive and time consuming and is limited to predesignated anatomical areas at the time of scanning, while isotope bone scans expose the patient to ionising radiation and are less pathologically specific.

Previous imaging studies using US ${ }^{15-18}$ have demonstrated the inaccuracy of clinical examination at detecting joint inflammation. Our study highlights that in this cohort of patients with early inflammatory arthritis, synovitis may occur in both painful (but not clinically synovitic joints) and asymptomatic joints. Arthroscopic evidence from patients with RA has previously shown that asymptomatic knee synovitis is common in patients with both active, untreated
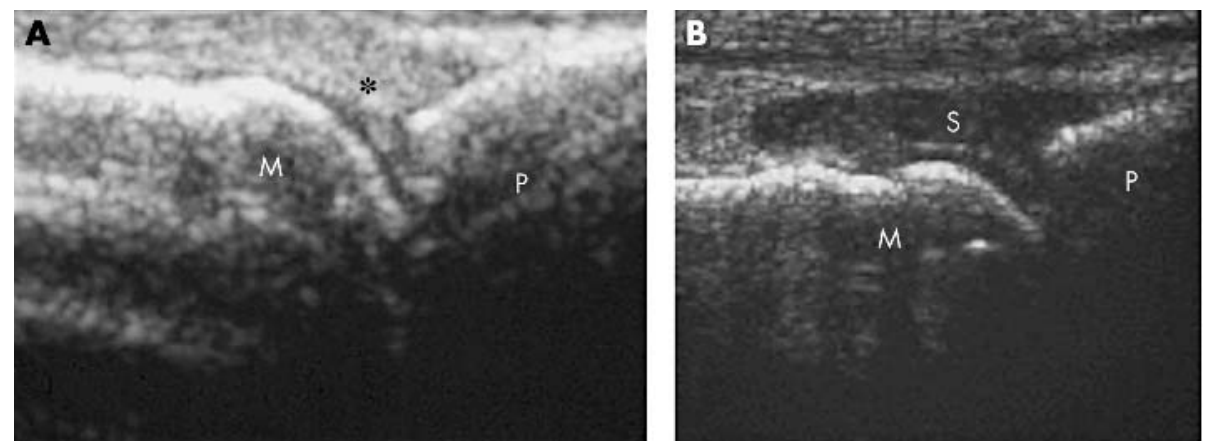

Figure 4 (A) Longitudinal US image through a non-painful MTP joint demonstrating no evidence of synovitis. $M$, metacarpal head; $P$, base of proximal phalanx; *normal intra-articular fat pad. (B) Longitudinal US image through a painful but not clinically synovitic MTP joint demonstrating synovitis (S) and loss of definition of normal intra-articular fat pad. $M$, metacarpal head; $P$, base of proximal phalanx. 
Table 3 Comparison of baseline diagnoses and proportion of patients with subclinical and polyarticular disease

\begin{tabular}{llll}
\hline Diagnosis & Number & $\begin{array}{l}\text { Subclinical } \\
\text { disease } \\
\text { No }(\%)\end{array}$ & $\begin{array}{l}\text { Polyarticular } \\
\text { disease } \\
\text { No (\%) }\end{array}$ \\
\hline Undifferentiated & 59 & $41(69)$ & $22(37)$ \\
oligoarthritis & 14 & $7(50)$ & $4(29)$ \\
Reactive arthritis & 14 & $3(50)$ & $2(33)$ \\
Psoriatic arthritis & 6 & 0 & 1 \\
Sarcoid & 1 & $51(64)$ & $29(36)$ \\
Total & 80 & & \\
\hline
\end{tabular}

early disease ${ }^{20}$ and chronic disease. ${ }^{21}$ In addition, Kraan et al have demonstrated in rhesus monkey models of RA that subclinical disease does precede clinical disease in prospective studies. ${ }^{22}$ This may account for the observation that many patients with RA already have signs of joint destruction by the time of presentation. ${ }^{23}$

Pando et al, with an arthroscopy study, suggested that patients with reactive arthritis were less likely to have subclinical disease than other forms of inflammatory arthritis. ${ }^{24}$ In our study, however, 14 patients had proven reactive arthritis at baseline; of these, $50 \%$ (7/14) had evidence of subclinical disease and 30\% had polyarticular disease. The different results by Pando et al may reflect smaller patient numbers or prior treatment with disease modifying drugs.

There are several limitations to our study. Firstly, we did not validate the additional synovitis by other imaging techniques-for example, arthroscopy, MRI, or isotope bone scanning. However, these techniques are limited by either an inability to scan more than one joint or a lack of specificity.

Secondly, we are aware that a healthy control group scanned randomly with the oligoarthritis patients would have strengthened the study. However, our own departmental data on asymptomatic hospital workers has demonstrated a low prevalence of synovitis-occurring in $<5 \%$ of MTP joints, MCP joints, and knees. Thirdly, the scanning process was time consuming taking about about 35 minutes/ patient (range 20-50). This may, however, have overestimated the time required as this included time for detailed documentation within the setting of a clinical study.

In summary, this study for the first time describes the US phenotype of patients with early, treatment naïve, "oligoarthritis". It highlights the relative insensitivity of routine clinical examination and demonstrates that subclinical synovitis as detected by US is common in these patients. A definition of oligoarthritis, therefore, based purely on clinical examination alone may be inappropriate and indicates that true oligoarthritis is a much rarer phenomenon. The findings of this study may have important implications for disease management by potentially allowing prediction of persistence and prognosis of those patients. Longitudinal assessment is, however, required to determine the true significance of subclinical disease.

\section{ACKNOWLEDGEMENTS}

We thank Sister Sue Burns, the local consultants for helping to recruit patients into the study, and Drs J Devlin and C Pease for reading the manuscript.

This study was funded by the Arthritis Research Campaign (UK). Professor Paul Emery is an ARC Professor in Rheumatology, Dr Richard Wakefield, Dr Michael Green, Dr Helena Marzo-Ortega, and
Dr Philip Conaghan have all been recipients of ARC funding, and Dr Dennis McGonagle is an MRC clinical scientist.

\section{Authors' affiliations}

R J Wakefield, M J Green, H Marzo-Ortega, P G Conaghan, D McGonagle, P Emery, Academic Department of Musculoskeletal Medicine, 1st Floor, Old Nurse's Home, General Infirmary at Leeds, Great George Street, Leeds LS1 3EX, UK

W W Gibbon, Department of Medical Imaging, Royal Brisbane Hospital, Herston Road, Herston, Brisbane, Queensland, Australia

S Proudman, Department of Rheumatology, Royal Adelaide Hospital, Adelaide, South Australia

\section{REFERENCES}

1 Hubscher O. Pattern recognition in arthritis. In: Hochberg M, Silman A, Smolen JS, Weinblatt ME, Weisman MH, eds. Rheumatology, 3rd ed. Sr Louis: Mosby, 2003.

2 Green M, Marzo-Ortega H, Wakefield RJ, Astin P, Proudman S, Conaghan PG, et al. Predictors of outcome in patients with oligoarthritis: results of a protocol of intra-articular corticosteroids to all clinically active joints. Arthritis Rheum $2001 ; 44: 1177-83$.

3 Wolfe F, Ross K, Hawley D, Roberts FK, Cathey MA. The prognosis of rheumatoid arthritis and undifferentiated polyarthritis syndrome in the clinic: a study of 1141 patients. J Rheumatol 1993;20:2005-9.

4 Kvein TK, Glennas A, Melby K. Prediction of diagnosis in acute and subacute oligoarthritis of unknown origin. Br J Rheumatol 1996;35:359-63.

5 Parker JD, Capell HA. An acute arthritis clinic-one year's experience. Br J Rheumatol 1986;25:293-5.

6 Manger B, Kalden J. Joint and connective tissue ultrasonography-a rheumatological bedside procedure? Arthritis Rheum 1995;38:736-42.

7 Balint P, Sturrock RD. Musculoskeletal ultrasound imaging: a new diagnostic tool for the rheumatologist? Br J Rheumatol 1997;36:1141-2.

8 Grassi W, Cervini C. Ultrasonography in rheumatology: an evolving technique. Ann Rheum Dis 1998;57:268-71.

9 Wakefield RJ, Gibbon WW, Emery P. The current status of ultrasonography in rheumatology. Rheumatology (Oxford) 1999;38:195-201.

10 Canosa JJ. Ultrasound imaging: a rheumatologists dream ? J Rheumatol 2000;27:2063

11 Grassi W, Filipucci E, Farina A, Shalaffi F, Cervini C. Ultrasonography for the evaluation of bone erosions. Ann Rheum Dis 2001;60:98-103.

12 Wakefield RJ, Gibbon WW, Conaghan PG, O'Connor P, McGonagle D, Pease $C P$, et al. The value of sonography in the detection of bone erosion in patients with rheumatoid arthritis: a comparative study with conventional radiography. Arthritis Rheum 2000;43:2762-70.

13 Backhaus M, Kamradt T, Sandrock D, Loreck D, Fritz J, Wolfe KJ, et al. Arthritis of the finger joints - a comprehensive approach comparing conventional radiography, scintigraphy, ultrasound, and contrast-enhanced magnetic resonance imaging. Arthritis Rheum 1999;42:232-45.

14 Alarcon GS, Lopez-Ben R, Moreland LW. High resolution ultrasound for the study of target joints in rheumatoid arthritis. Arthritis Rheum 2002;46:1969-81.

15 Jacobson JA, Andresen R, Jaovisidha S, De Maeseneer M, Foldes K, Trudell DR, et al. Detection of ankle effusions: comparison study in cadavers using radiography, sonography, and MR imaging. AJR 1998;170:1231-8.

16 Schmidt WA, Volker L, Zacher J, Schalfke M, Ruhnke M, Gromnica-Ihle E. Colour Doppler ultrasonography to detect pannus in knee joint synovitis. Clin Exp Rheumatol 2000;18:439-44.

17 Koski J. Ultrasonography of the metatarsophalangeal and talocrural joints. Clin Exp Rheumatol 1990;8:347-51.

18 Van Holsbeeck M, Van Holsbeeck K, Gevers G, Marchal G, Van Steen A, Favril A, et al. Staging and Follow-up of rheumatoid arthritis of the knee: comparison of sonography, thermography, and clinical assessment. J Ultrasound Med 1988;7:561-6.

19 Backhaus M, Gerber T, Grassi W, Machold K, Swen WA, Wakefield RJ, et al. Guidelines for musculoskeletal ultrasound in rheumatology: a review. Ann Rheum Dis 2001;60:641-9.

20 Reece RJ, Green MJ, McGonagle D, Proudman S, Kinnon J, Veale D, et al. Arthroscopic determination of subclinical knee synovitis in early rheumatoid arthritis [abstract]. Br J Rheumatol 1997;36(suppl):S89.

21 Sodden M, Rooney M, Cullen A, Whelan A, Feighery C, Bresnihan B. Immunohistological features in the synovium obtained from clinically uninvolved knee joints of patients with rheumatoid arthritis. $\mathrm{Br} J$ Rheumatol 1989;28:287-92.

22 Kraan MC, Versendaal H, Jonker M, Bresnihan B, Post WJ, Hart BA, et al. Asymptomatic synovitis precedes clinically manifest arthritis. Arthritis Rheum 1998;41:1481-8.

23 Van der Heijde DM. Joint erosions and patients with early rheumatoid arthritis. BrJ Rheumatol 1995;34:74-8.

24 Pando JA, Duray P, Yarboro C, Gourley MF, Klippel JH, Schumacher HR. Synovitis occurs in some clinically normal and asymptomatic joints in patients with early arthritis. J Rheumatol 2000;27:1848-54. 